\title{
Stiffness and damping characteristics of conical multirecess hybrid journal bearing for different load arrangements
}

\author{
N. K. Rana ${ }^{1}$. S. S. Gautam ${ }^{1}$ - S. Verma ${ }^{2}$
}

(C) Springer Nature Switzerland AG 2019

\begin{abstract}
This paper presents the theoretical analysis on the effect of two different load arrangements for a conical multirecess hybrid journal bearing. For this analysis constant flow valve restrictors are used under micropolar lubricant for different semi cone angles. The modified Reynolds equation using micropolar fluid flow on the conical surface has been solved with the use of FEM and Galerkin's technique applying proper boundary conditions in the iterative scheme. The study suggests that the configuration LA I can be a better alternative as compared to LA II for different semi-cone angles and external load in terms of bearing characteristics.
\end{abstract}

Keywords CFV restrictor · Conical bearing $\cdot$ Micropolar lubrication · FEM

\section{List of symbols}

$a_{b}$

$A_{p}$

$A_{b}$

$A^{e}$

$C$

$C_{i j}$

$D_{m}$

e

$F$

$F_{x}, F_{z}$

$h$

$I_{m}, \bar{I}$

$L$

$M$

$p_{s}$

$Q$

$R_{j}$

$r$

$S_{i j}$

$\omega_{j}$
Axial bearing land width, $\mathrm{mm}$

Area of pocket, $\mathrm{mm}^{2}$

Area of bearing, $\mathrm{mm}^{2}$

Area of each element

Radial clearance, $\mathrm{mm}$

Fluid film damping coefficients $\left(\mathrm{N} / \mathrm{mm}^{2}\right)$,

$(i, j=x, z)$

Mean journal diameter of conical shaft, $\mathrm{mm}$

Eccentricity, $\mathrm{mm}$

Fluid film reaction

Fluid film reaction component, $\mathrm{N}$

Fluid film thickness, $\mathrm{mm}$

Bearing characteristic length

Bearing length, $\mathrm{mm}$

Journal mass, $\mathrm{kg}$

Supply pressure, $\mathrm{N} / \mathrm{mm}^{2}$

Lubricant flow $\left(\mathrm{mm}^{3} / \mathrm{s}\right)$

Journal radius, $\mathrm{mm}$

Radial coordinate

Fluid film stiffness coefficients ( $\mathrm{N} / \mathrm{mm})$,

$(i, j=x, z)$

Journal rotation speed, $\mathrm{rad} / \mathrm{s}$ $\omega_{\text {th }} \quad$ Threshold speed, rad/s

$W_{r} \quad$ Radial load, N

$X_{j}, Z_{j} \quad$ Journal center coordinate

$x, y, z \quad$ Cartesian coordinate system

\section{Greek symbol}

$\alpha \quad$ Circumferential coordinate

$\beta \quad$ Axial co-ordinate

$\gamma \quad$ Semi cone angle, degree

$\mu \quad$ Dynamic viscosity $\left(\mathrm{P}_{a} s\right)$

$\lambda \quad$ Aspect ratio, $L / D_{m}$

$\theta \quad$ Inter-recess angle

$\Omega \quad$ Speed parameter

$\varepsilon \quad$ Eccentricity ratio $e / c$

$\emptyset \quad$ Micro polar function

$\varphi \quad$ Attitude angle

Non-dimensional number/parameter

$\bar{a}_{b} \quad a_{b} / L$

$\bar{C}_{i j} \quad C_{i j}\left(c^{3} / \mu r_{j}^{4}\right)$

$\bar{F}_{r} \quad\left(F / p_{s}\right)$

$\bar{F}_{x}, \bar{F}_{z} \quad\left(F_{x}, F_{z} / p_{s} r_{j}^{2}\right)$

$\triangle$ S. S. Gautam, ssg@nerist.ac.in | ${ }^{1}$ Mechanical Engineering Department, North Eastern Regional Institute of Science and Technology, Itanagar, Arunachal Pradesh 791109, India. ${ }^{2}$ Mechanical Engineering Department, Deenbandhu Chhotu Ram University of Science and Technology, Murthal, Sonepat, Haryana 131039, India. 
$\bar{F}_{0} \quad$ Resultant fluid film force at steady state condition

$\bar{h}_{\min }$

$\bar{P}_{\max }^{\min }$ Minimum fluid film thickness

$\bar{M}_{c}$

$N^{2}$

$N_{i}, N_{j}$

$\bar{P}_{j}$

$\bar{Q}$

$\bar{Q}_{c}$

$\bar{S}_{i j}$

$\bar{\omega}_{t h}$

$\overline{\dot{X}}_{j}, \overline{\mathrm{Z}}_{j}$ Maximum fluid film pressure

$M\left(c g / p_{s} R_{j}^{3}\right)$

Coupling number

Shape function

$\left(p / p_{s}\right)$

$Q\left(\mu / c^{3} p_{s}\right) r$

Constant flow valve restrictor design parameter

$S_{i j}\left(c / p_{s} r_{j}^{2}\right)$

$\quad \sqrt{\bar{M}_{c} / \bar{F}_{0}}$

Matrices

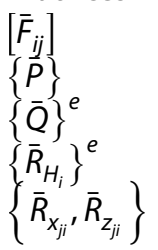

Fluidity matrix

Nodal pressure vector

Nodal flow vector

Column vector due to hydrodynamic term

Nodal R.H.S vector due to journal center

velocity

\section{Introduction}

Conical journal bearings offer the advantages over cylindrical bearings. They can support radial as well as thrust load thereby can replace two bearings with one and they have adjustable radial clearance during assembly. However, these advantages are at the expense of corresponding requirement of accuracy level in machining of surfaces and accurate assembly of conical bearing. Many researchers have carried out the theoretical study on the conical bearing geometry. The multirecess externally pressurised journal bearing with micropolar lubrication has been studied by Verma et al. [1]. Rana et al. have studied theoretically the effect of micropolar fluid parameters on the steady state as well as stiffness and damping characteristics of 4-pocket constant flow valve compensated hydrostatic conical journal bearing [2, 3]. Sharma et al. [4, 5] theoretically analyzed the effect of wear on the characteristics of a 4 pocket hydrostatic/hybrid conical journal bearing system for different cone angles with different restrictors for different load arrangements. The analysis of multirecessed hybrid conical journal bearing with various restrictors have been carried out by Rajput and Sharma $[6,7]$. Dhawan and Verma [8] have studied non-circular hybrid journal bearings with micropolar fluid film lubricant. Sharma et al. [9] carried out the performance characteristics of multirecess hydrostatic/hybrid flexible journal bearing compensated with membrane type variable flow restrictor. Performance analysis of a hybrid conical journal bearing with capillary restrictors has been presented by Khakse et al. [10]. Guo et al. [11] studied the performance of hydrostatic deep/shallow pocket hybrid conical bearing compensated by capillary and seen that the stability and load carrying capacity improves under small eccentric operation. Zuo et al. [12] have analyzed the comparative performance of conical hydrostatic bearings compensated by variable slot and fixed slot. They have concluded that variable slot compensated bearing exhibits a better radial load carrying capacity as well as the stiffness coefficients. The stability of the rigid rotor supported by six recessed hybrid bearings with orifice compensation and influence of restrictor design parameters and eccentricity on it are studied by Chen et al. [13]. Rana et al. [14, 15] have analyzed the performance of four and six pockets conical hybrid journal bearing.

The performances of conical hybrid journal bearing with constant flow valve for micropolar lubricant for different load arrangements have not been exploited by the researchers. Hence, the efforts have been made to
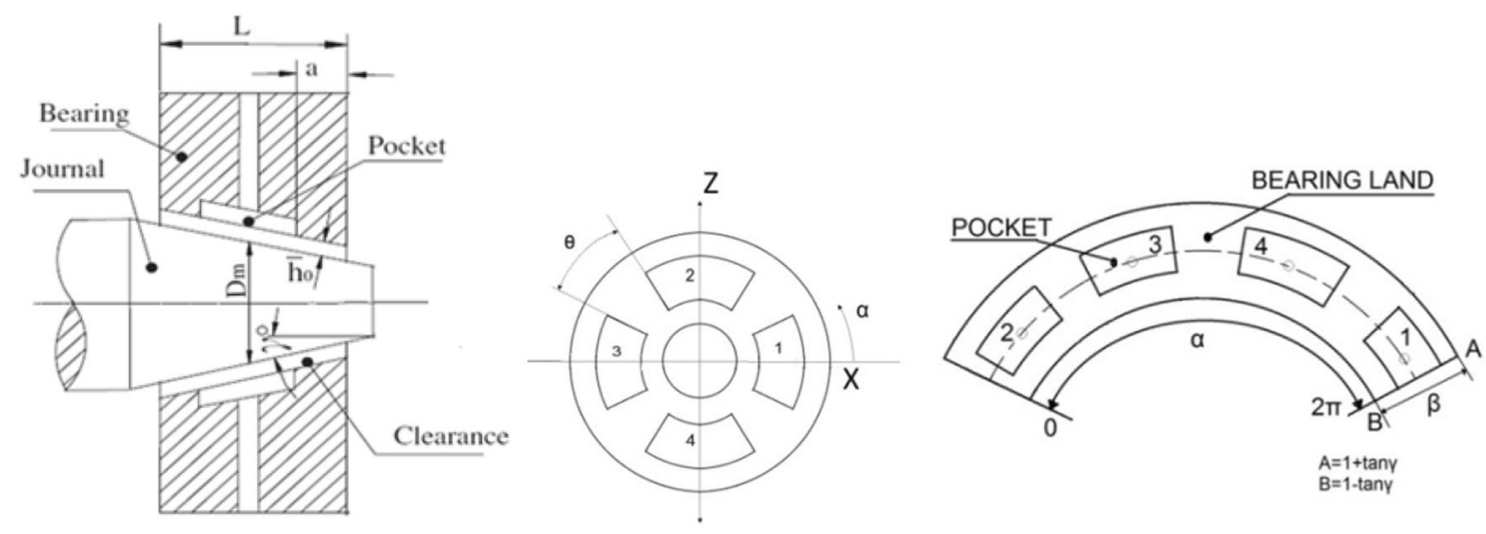

Fig. 1 Conical hybrid journal bearing [14]

SN Applied Sciences

A SPRINGER NATURE journal 
highlight this issue in the present work to analyze the bearing performances. Based on the above fact, the CFV compensated conical hybrid journal bearing performance for different loading conditions LA I (i.e. load line in between the two recesses) and LA II (i.e. load line on a recess) under micropolar lubrication has been carried out. The data charts presented here for multirecess hybrid bearings may be useful for the bearing designers as a guide for bearing performance.

\subsection{Governing equation}

The 4-pocket hybrid conical journal bearing configurations are shown in Figs. 1 and 2 with journal assuming equilibrium position under rotation with uniform angular velocity.

For conical surface using micropolar fluid the Reynolds equation in non-dimensional form is as follows [5-7]:

$\frac{1}{\beta^{2}} \frac{\partial}{\partial \alpha}\left[\frac{\bar{\emptyset}(N, \bar{l}, \bar{h})}{12} \frac{\partial \bar{p}}{\partial \alpha}\right]+\frac{\sin ^{2} \gamma}{\beta} \frac{\partial}{\partial \beta}\left[\frac{\beta \bar{\emptyset}(N, \bar{l}, \bar{h})}{12} \frac{\partial \bar{p}}{\partial \beta}\right]=\frac{\Omega}{2} \frac{\partial \bar{h}}{\partial \alpha}+\frac{\partial \bar{h}}{\partial \bar{t}}$

where

$\beta=\frac{r \sin \gamma}{R_{j}}, \bar{p}=\frac{p}{p_{s}}, \bar{h}=\frac{h}{C}, \bar{t}=t\left(\frac{C^{2} p_{s}}{R_{j}^{2} \mu}\right), \bar{l}=\frac{c}{l_{m}}$

$\bar{\emptyset}(N, \bar{l}, \bar{h})=1+\frac{12}{\bar{h}^{2} \bar{l}^{2}}-\frac{6 N}{\bar{h} \bar{l}} \operatorname{coth}\left(\frac{N \bar{h} \bar{l}}{2}\right)$

\subsection{Finite element formulation}

The governing element equation is obtained with the help of Galerkin's technique along with the orthogonality conditions as well as approximate value of non-dimensional pressure. Therefore, Eq. (1) can be expressed as follows:

$$
\begin{aligned}
& \iint_{A^{e}}\left\{\frac{\sin ^{2} \gamma}{\beta} \frac{\partial}{\partial \beta}\left[\frac{\beta \bar{\emptyset}(N, \bar{l}, \bar{h})}{12} \frac{\partial \bar{p}}{\partial \beta}\right]+\frac{1}{\beta^{2}} \frac{\partial}{\partial \alpha}\left[\frac{\bar{\emptyset}(N, \bar{l}, \bar{h})}{12} \frac{\partial \bar{p}}{\partial \alpha}\right]\right. \\
& \left.-\frac{\Omega}{2} \frac{\partial \bar{h}}{\partial \alpha}-\frac{\partial \bar{h}}{\partial \bar{t}}\right\} N_{j} d \alpha d \beta=0 \\
& \iint_{A^{e}}\left\{\frac{\sin ^{2} \gamma}{\beta} \frac{\partial}{\partial \beta}\left[\frac{\beta \bar{\emptyset}(N, \bar{l}, \bar{h})}{12} \frac{\partial \bar{p}}{\partial \beta}\right]+\frac{1}{\beta^{2}} \frac{\partial}{\partial \alpha}\left[\frac{\bar{\emptyset}(N, \bar{l}, \bar{h})}{12} \frac{\partial \bar{p}}{\partial \alpha}\right]\right. \\
& \left.-\frac{\Omega}{2} \frac{\partial \bar{h}}{\partial \alpha}+\bar{X}_{j} \cos \alpha \cos \gamma+\overline{\dot{Z}}_{j} \sin \alpha \cos \gamma\right\} N_{j} d \alpha d \beta=0
\end{aligned}
$$

This Eq. (2) is intergrated part wise and combined by multiplying with the term $\frac{\beta^{2}}{\sin \gamma}$, which transforms as,

$$
\begin{aligned}
& \iint_{A^{e}} \frac{\bar{\emptyset}\left(N_{,} \bar{l}, \bar{h}\right)}{12}\left[\beta^{2} \sin \gamma \frac{\partial N_{i}}{\partial \beta} \frac{\partial N_{j}}{\partial \beta}+\frac{1}{\sin \gamma} \frac{\partial N_{i}}{\partial \alpha} \frac{\partial N_{j}}{\partial \alpha}\right] \bar{p}_{j} d \alpha d \beta \\
& \quad+\int_{\Gamma^{e}} \frac{\bar{\emptyset}(N, \bar{l}, \bar{h})}{12}\left[\frac{1}{\sin \gamma} \frac{\partial \bar{p}}{\partial \alpha} I_{1}+\beta^{2} \sin \gamma \frac{\partial \bar{p}}{\partial \beta} I_{2}\right] N_{i} d \Gamma^{e} \\
& \quad-\frac{\Omega}{2} \int_{\Gamma^{e}} N_{i} \frac{\beta^{2}}{\sin \gamma} \bar{h} l_{1} d \Gamma^{e}+\frac{\Omega}{2} \iint_{A^{e}} \bar{h} \frac{\beta^{2}}{\sin \gamma} \frac{\partial N_{i}}{\partial \alpha} d \alpha d \beta \\
& +\iint_{A^{e}} \bar{X}_{j} \frac{\beta^{2}}{\sin \gamma} \cos \alpha \cos \gamma N_{i} d \alpha d \beta \\
& +\iint_{A^{e}} \bar{Z}_{j} \frac{\beta^{2}}{\sin \gamma} \sin \alpha \cos \gamma N_{i} d \alpha d \beta=0
\end{aligned}
$$

(a) LA I

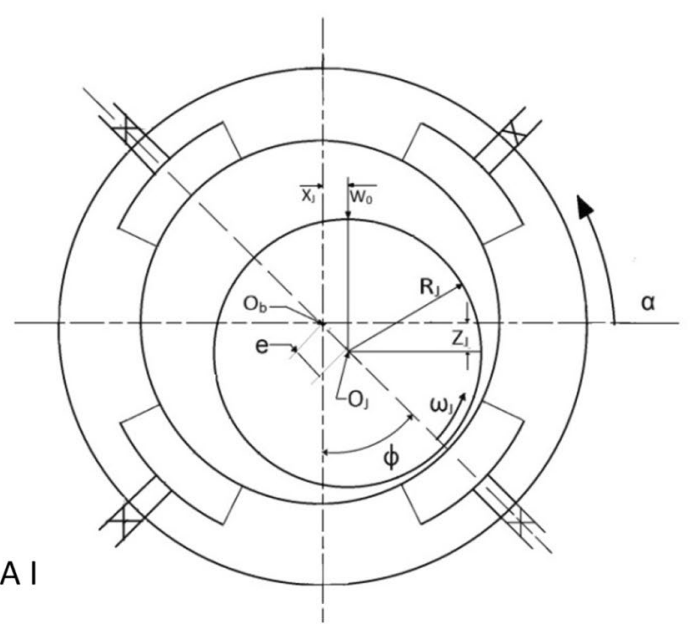

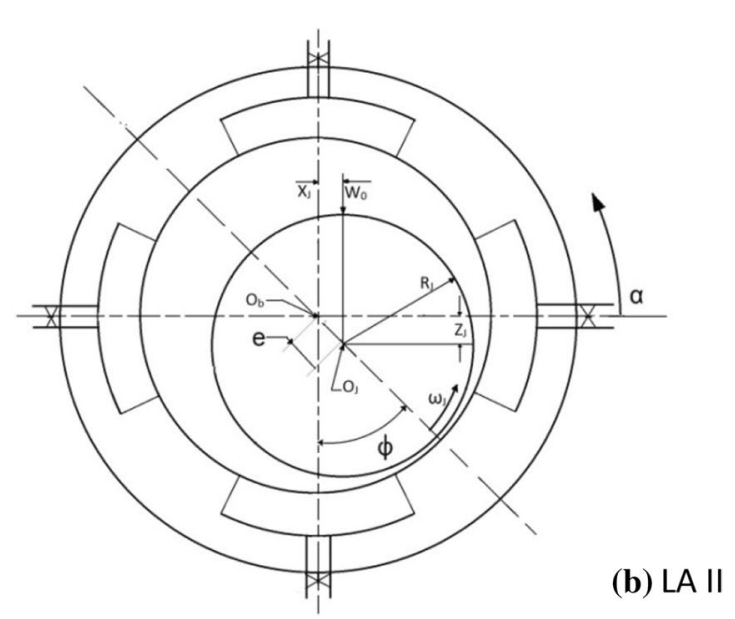

Fig. 2 LA I and LA II configurations of conical hybrid journal bearing 

as:

Now the above equation is represented in matrix form

$\left[\bar{F}_{i j}\right]\{\bar{p}\}=\{\bar{Q}\}^{e}+\Omega\left\{\bar{R}_{H_{i}}\right\}^{e}+\bar{X}_{j}\left\{\bar{R}_{x_{j i}}\right\}^{e}+\overline{\dot{Z}}_{j}\left\{\bar{R}_{z_{j i}}\right\}^{e}$

where

$$
\begin{aligned}
{\left[\bar{F}_{i j}\right]=} & \iint_{A^{e}} \frac{\bar{\emptyset}(N, \bar{l}, \bar{h})}{12}\left[\frac{1}{\sin \gamma} \frac{\partial N_{i}}{\partial \alpha} \frac{\partial N_{j}}{\partial \alpha}+\beta^{2} \sin \gamma \frac{\partial N_{i}}{\partial \beta} \frac{\partial N_{j}}{\partial \beta}\right] d \alpha d \beta \\
\left\{\bar{Q}_{i}\right\}^{e}= & \int_{\Gamma^{e}} \frac{\bar{\emptyset}(N, \bar{l}, \bar{h})}{12}\left[\frac{1}{\sin \gamma} \frac{\partial \bar{p}}{\partial \alpha} I_{1}+\beta^{2} \sin \gamma \frac{\partial \bar{p}}{\partial \beta} I_{2}\right] N_{i} d \Gamma^{e} \\
& -\frac{\Omega}{2} \int_{\Gamma^{e}} \frac{\beta^{2}}{\sin \gamma} \bar{h} l_{1} N_{i} d \Gamma^{e}
\end{aligned}
$$$$
\bar{R}_{H_{i}}^{e}=\iint_{A^{e}} \frac{\bar{h}}{2} \frac{\partial N_{i}}{\partial \alpha} \frac{\beta^{2}}{\sin \gamma} d \alpha d \beta
$$$$
\bar{R}_{X_{j i}}^{e}=\iint_{A^{e}} N_{i} \cos \alpha \cos \gamma \frac{\beta^{2}}{\sin \gamma} d \alpha d \beta
$$$$
\bar{R}_{Z_{j i}}^{e}=\iint_{A^{e}} N_{i} \sin \alpha \cos \gamma \frac{\beta^{2}}{\sin \gamma} d \alpha d \beta
$$

\subsection{Stiffness and damping coefficients}

The stiffness coefficients for fluid film in bearing are expressed as

$$
\left[\begin{array}{ll}
\bar{S}_{x x} & \bar{S}_{x z} \\
\bar{S}_{z x} & \bar{S}_{z z}
\end{array}\right]=-\left[\begin{array}{lll}
\partial \bar{F}_{x} / \partial \bar{X}_{j} & \partial \bar{F}_{x} / \partial \bar{Z}_{j} \\
\partial \bar{F}_{z} / \partial \bar{X}_{j} & \partial \bar{F}_{z} / \partial \bar{Z}_{j}
\end{array}\right]
$$

and the damping coefficients are expressed as

$$
\left[\begin{array}{ll}
\bar{C}_{x x} & \bar{C}_{x z} \\
\bar{C}_{z x} & \bar{C}_{z z}
\end{array}\right]=-\left[\begin{array}{ll}
\partial \bar{F}_{x} / \partial \overline{\dot{X}}_{j} & \partial \bar{F}_{x} / \partial \overline{\dot{Z}}_{j} \\
\partial \bar{F}_{z} / \partial \overline{\dot{X}}_{j} & \partial \bar{F}_{z} / \partial \overline{\dot{Z}}_{j}
\end{array}\right]
$$

\subsection{Stability parameters}

Equation for non-dimensional critical mass, $\bar{M}_{c}$

$\bar{M}_{c}=\frac{\bar{G}_{1}}{\bar{G}_{2}-\bar{G}_{3}}$

$$
\bar{G}_{1}=\left[\bar{C}_{x x} \bar{C}_{z z}-\bar{C}_{z x} \bar{C}_{x z}\right]
$$

$\bar{G}_{2}=\frac{\left[\bar{S}_{x x} \bar{S}_{z z}-\bar{S}_{z x} \bar{S}_{x z}\right]\left[\bar{C}_{x x}+\bar{C}_{z z}\right]}{\left[\bar{S}_{x x} \bar{C}_{z z}+\bar{S}_{z z} \bar{C}_{x x}-\bar{S}_{x z} \bar{C}_{z x}-\bar{S}_{z x} \bar{C}_{x z}\right]}$

$\bar{G}_{3}=\frac{\left[\bar{S}_{x x} \bar{C}_{x x}+\bar{S}_{x z} \bar{C}_{x z}+\bar{S}_{z x} \bar{C}_{z x}+\bar{S}_{z z} \bar{C}_{z z}\right]}{\bar{C}_{x x}+\bar{C}_{z z}}$

Equation for threshold speed, $\bar{\omega}_{\text {th }}$

$\bar{\omega}_{t h}=\sqrt{\frac{\bar{M}_{c}}{\bar{F}_{0}}}$

\subsection{Fluid film thickness}

For conical journal bearing, the fluid film thickness can be expressed in non-dimensional form as,

$\bar{h}=\left(1-\bar{X}_{j} \cos \alpha-\bar{Z}_{j} \sin \alpha\right) \cos \gamma$

\subsection{Restrictor flow equation}

The flow control device used here is CFV restrictor. The lubricant supply passing through it must be a fixed quantity. The lubricant flow $\bar{Q}_{R}$ can be written as

$\bar{Q}_{R}=$ constant $=\bar{Q}_{c}$

here $\bar{Q}_{R}$ and $\bar{Q}_{c}$ areflow through restrictor and pocket, respectively.

\subsection{Boundary conditions}

1. Lubricant flow through restrictor equals input flow of the bearing $\left(\bar{Q}_{R}=\bar{Q}_{c}\right)$.

2. All the nodes situated on a pocket/recess have the same pressure.

3. The nodes situated at the edges of bearing have atmospheric pressure i.e. zero gauge pressure $\left(\left.\bar{p}\right|_{\beta=\mp 1.0}=0.0\right)$.

4. It is assumed that Reynolds boundary condition is applicable at the trailing portion of positive pressure region i.e.

$$
\bar{p}=\frac{\partial \bar{p}}{\partial \alpha}=0.0
$$

In the present study the Reynolds boundary condition is used due to absence of rupture and reformation of the lubricant film. 


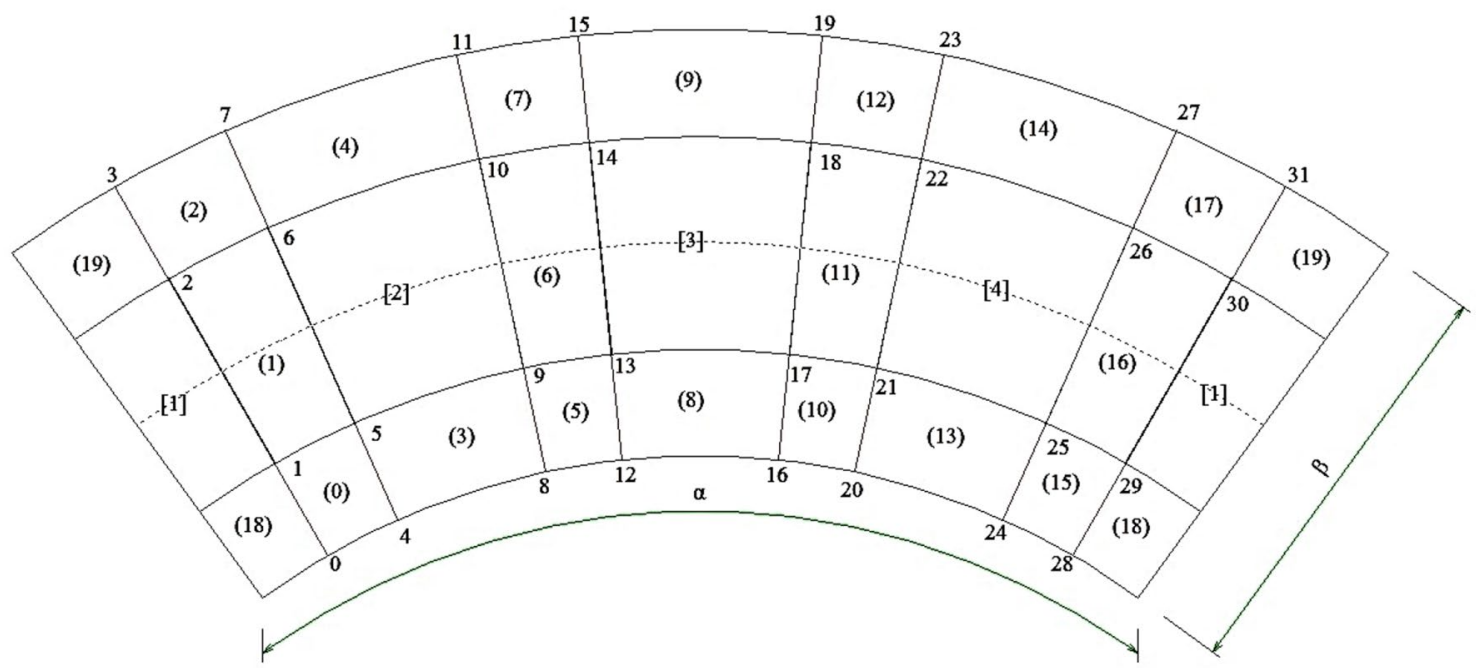

Fig. 3 Discretized 4-pocket conical journal bearing [14]

\subsection{Solution procedure}

The discretization of flow field of micropolar fluid in the lubrication zone of the conical journal bearing system using 4 noded isoparametric elements and Langragian interpolation function is done. The pressure is distributed in the element of discretized zone and can be expressed for 4 pockets by:

$\bar{p}=\sum_{i=1}^{4} N_{j} \bar{P}_{j}$

The 2-dimensional mesh of 4 pockets conical journal bearing with nodes as well as elements are shown in Fig. 3.

The governing Reynolds equation, which governs the micropolar fluid, is solved taking into consideration of restrictor flow and suitable boundary conditions by finite element technique to obtain the fluid film pressures. The iterations are repeated till solutions are converged for calculation of fluid film pressure.

The computations for the steady state characteristics of hybrid journal bearing system requires input data file. The INPDAT module reads input data for 2-D meshing. The fluid film thickness for different nodal points and tentative journal center positions is calculated by the subroutine FLDTHK. The fluidity matrix is created and generalized according to connectivity of various elements through subroutine FEQN. The boundary condition of the system is specified by the CALL_BOUNDARY subroutine. The governing Reynolds equation for conical bearing is solved using Gaussian elimination procedure for the calculation of fluid film pressures at different nodes through subroutine CALL_SOLVER. The load carrying limits are calculated as well. The journal center and its equilibrium position are ascertained following iterations shown in solution scheme by JCE with the use of $\bar{F}_{x}$ and $\bar{F}_{z}$ components. Finally, the SPC subroutine calculates the steady state parameters of the conical bearing that can be seen in Fig. 12 ("Appendix").

\subsection{Discussion of results}

The mathematical model and the solution scheme presented in the previous section are used to calculate the bearing performances and stability parameters of CFV compensated 4-pocket conical hybrid journal bearing under micropolar lubrication. The hybrid journal bearing must sustain the operating load at maximum speed and the partial load at zero speed respectively. The maximum speed considered in this analysis is $\Omega=1.0$ for all data charts. The values are computed for the following non-dimensional data of the bearing and geometric parameters:

$\Omega=1.0, \lambda=1.0, \bar{a}_{b}=0.25, \bar{W}_{r}=0.5, \bar{Q}_{c}=0.1-0.3, \theta=18^{\circ}$,

$$
A_{p} / A_{b}=0.333, N^{2}=0.3-0.9, \bar{l}=10-30, \gamma=10^{\circ}-40^{\circ}
$$

To build confidence in the results, the validity of the formulation of Reynolds equation and its solution scheme developed is established by employing the results available in literature for different cases. The results obtained are compared with the data of Sharma and Rajput [6] and are shown in Table 1. The compared results are seen to be in close agreement with the published data. The various performance characteristics for Newtonian and micropolar fluid parameters for load arrangements I \& II are presented in Table 2. 
Table 1 Validation for conical bearing with capillary: $\lambda=1.0$, $\Omega=1.0, \bar{W}_{r}=0.5, C_{s 2}=0.5$, $A_{p} / A_{b}=0.333, \gamma=10^{0}$

\begin{tabular}{|c|c|c|c|c|c|c|}
\hline$L_{M}$ & $N^{2}$ & Comparison & $\bar{P}_{\max }$ & $\bar{h}_{\min }$ & $\bar{Q}$ & $\varphi$ \\
\hline \multirow[t]{4}{*}{10} & 0.4 & Present result & 0.6747 & 0.8953 & 0.8464 & 61.77 \\
\hline & 0.4 & Sharma and Rajput [6] & 0.6796 & 0.8884 & 0.8522 & 61.00 \\
\hline & 0.5 & Present result & 0.6898 & 0.8981 & 0.8161 & 62.10 \\
\hline & 0.5 & Sharma and Rajput [6] & 0.6947 & 0.8914 & 0.8218 & 61.00 \\
\hline \multirow[t]{4}{*}{20} & 0.4 & Present result & 0.6436 & 0.8898 & 0.9096 & 60.40 \\
\hline & 0.4 & Sharma and Rajput [6] & 0.6485 & 0.8825 & 0.9156 & 59.00 \\
\hline & 0.5 & Present result & 0.6503 & 0.8910 & 0.8960 & 60.52 \\
\hline & 0.5 & Sharma and Rajput [6] & 0.6553 & 0.8840 & 0.9020 & 59.00 \\
\hline \multirow[t]{4}{*}{30} & 0.4 & Present result & 0.6302 & 0.8870 & 0.9366 & 60.02 \\
\hline & 0.4 & Sharma and Rajput [6] & 0.6352 & 0.8795 & 0.9426 & 59.00 \\
\hline & 0.5 & Present result & 0.6344 & 0.8879 & 0.9281 & 60.07 \\
\hline & 0.5 & Sharma and Rajput [6] & 0.6394 & 0.8805 & 0.9341 & 59.00 \\
\hline
\end{tabular}

Table 2 Comparisons of Newtonian and micropolar fluid in terms of different bearing characteristics at $\Omega=1, \bar{Q}_{c}=0.2, \bar{W}_{r}=0.5, \bar{a}=0.25, \lambda=1, \gamma=20^{\circ}$

\begin{tabular}{|c|c|c|c|c|c|c|c|c|c|c|}
\hline & \multicolumn{2}{|c|}{ Newtonian } & \multicolumn{2}{|c|}{$N^{2}=0.3, \bar{I}=10$} & \multicolumn{2}{|c|}{$N^{2}=0.9, \bar{I}=10$} & \multicolumn{2}{|c|}{$N^{2}=0.3, \bar{I}=30$} & \multicolumn{2}{|c|}{$N^{2}=0.9, \bar{I}=30$} \\
\hline & LA I & LA II & LA I & LA II & LA I & LA II & LA I & LA II & LA I & LA II \\
\hline $\bar{h}_{\min }$ & 0.745 & 0.709 & 0.778 & 0.733 & 0.803 & 0.766 & 0.764 & 0.721 & 0.774 & 0.731 \\
\hline $\bar{p}_{\max }$ & 0.493 & 0.560 & 0.566 & 0.632 & 0.693 & 0.758 & 0.524 & 0.591 & 0.555 & 0.621 \\
\hline $\bar{S}_{11}$ & 7.004 & 4.274 & 8.297 & 5.208 & 11.102 & 7.114 & 7.609 & 4.682 & 8.241 & 5.099 \\
\hline $\bar{S}_{22}$ & 3.280 & 7.823 & 4.090 & 9.287 & 5.833 & 12.42 & 3.64 & 8.507 & 4.013 & 9.213 \\
\hline $\bar{C}_{11}$ & 4.944 & 4.067 & 6.118 & 4.913 & 8.329 & 6.548 & 5.428 & 4.415 & 5.909 & 4.765 \\
\hline $\bar{C}_{22}$ & 3.342 & 2.394 & 4.078 & 3.092 & 5.517 & 4.398 & 3.645 & 2.684 & 3.951 & 2.971 \\
\hline
\end{tabular}

\subsubsection{Influence on minimum fluid film thickness $\left(\bar{h}_{\text {min }}\right)$ and maximum fluid film pressure $\left(\bar{p}_{\text {max }}\right)$}

Here the numerical analysis has been performed for the recessed hybrid conical bearing, having symmetrically placed 4-pockets, for two possible loading arrangements namely LA I and LA II. In LA I, the vertical downward load line passes between the two recesses i.e. through landwidth of bearing while in LA II arrangement, the load line passes through the recess in the bearing and is the conventional arrangement used in the bearing design.

The plot of minimum fluid film thickness $\bar{h}_{\min }$ with $\bar{W}_{r}$ is shown in Fig. 4. The value of $\bar{h}_{\min }$ decreases with an increase in $\bar{W}_{r}$ for different angles $\gamma$ and for different loading conditions LA I and LA II. It is further noticed that $\bar{h}_{\text {min }}$ is minimum for LA II than LA I for $\gamma=20^{\circ}$ and $\gamma=40^{\circ}$ for the same operating and geometric conditions considered. As the $\bar{h}_{\text {min }}$ for LA I is more as compared to LA II, so it can accommodate a significant amount of surface worn and surface roughness.
The plot of $\bar{p}_{\max }$ versus $\bar{W}_{r}$ at $\Omega=1$ for LA I \& LA II are shown in Fig. 5 . The value of $\bar{p}_{\max }$ increases with an increase in $\bar{W}_{r}$ and the trend is same for both the loading conditions. Also, as the maximum fluid film pressure is reciprocal to minimum fluid film thickness, the $\bar{p}_{\max }$ is seen to be higher for LA II when compared to LA I for the same value of $\bar{W}_{r}$ considered in the range of 0.1 to 0.5 . Whereas, LA I could have been a better alternative to reduce the $\bar{p}_{\text {max }}$ for the same $\bar{W}_{r}$.

\subsubsection{Influence on stiffness coefficients $\left(S_{i j}\right)$ and damping coefficients $\left(C_{i j}\right)$}

The plots of direct stiffnesses coefficient versus $\bar{W}_{r}$ are given in Figs. 6 and 7. The stiffness coefficients $\left(\bar{S}_{11}\right)$ and $\left(\bar{S}_{22}\right)$ remains almost same with an increase in $\bar{W}_{r}$ for LA I and LA II as well as for different semi cone angles. The value of $\bar{S}_{11}$ is seen to be higher for LA I position as shown in Fig. 6. Whereas, the value of stiffness coefficient $\left(\bar{S}_{22}\right)$ is higher for LA II position in comparison to LA I. The 


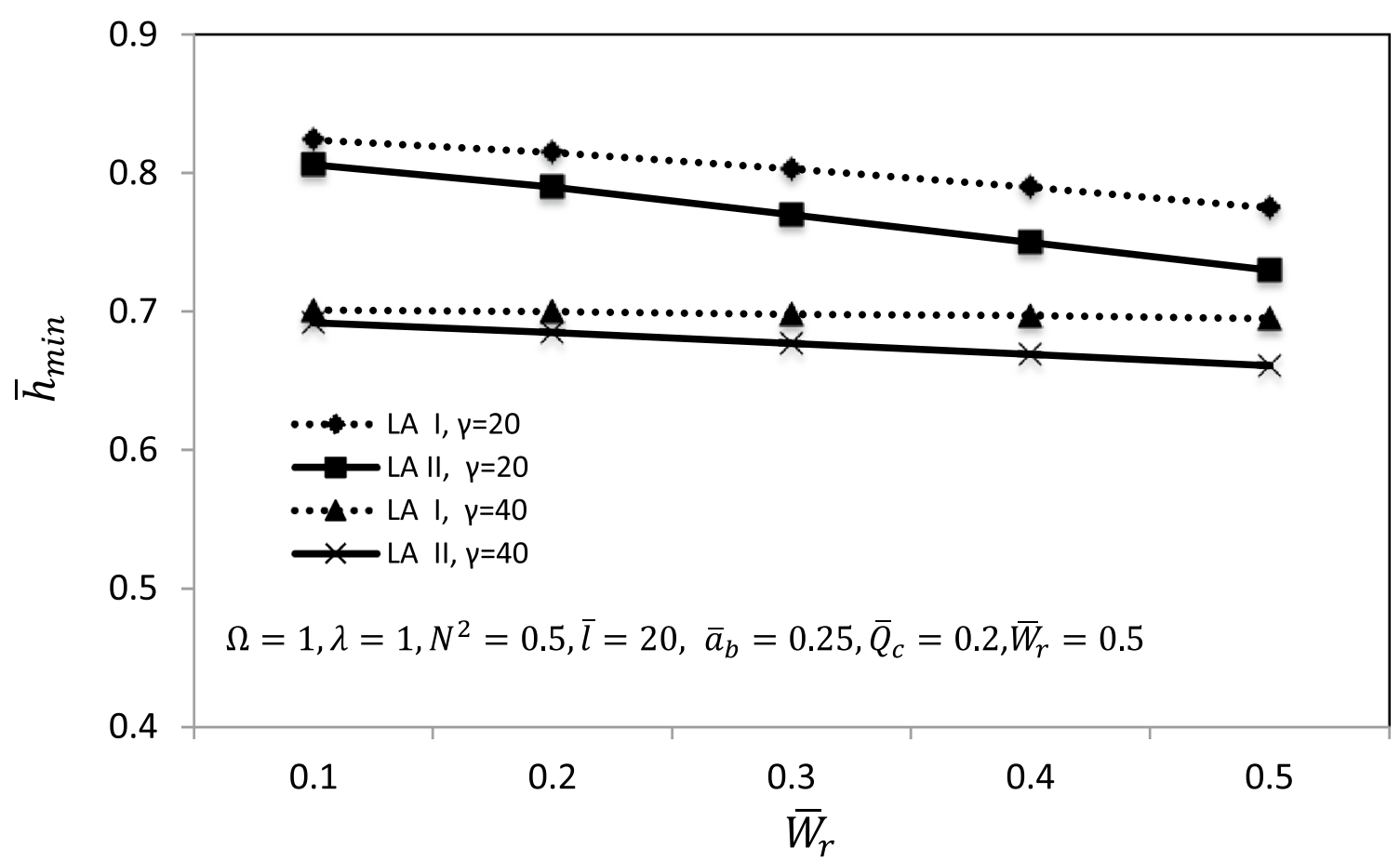

Fig. 4 Minimum fluid film thickness $\left(\bar{h}_{\min }\right)$ versus radial $\operatorname{load}\left(\bar{W}_{r}\right)$

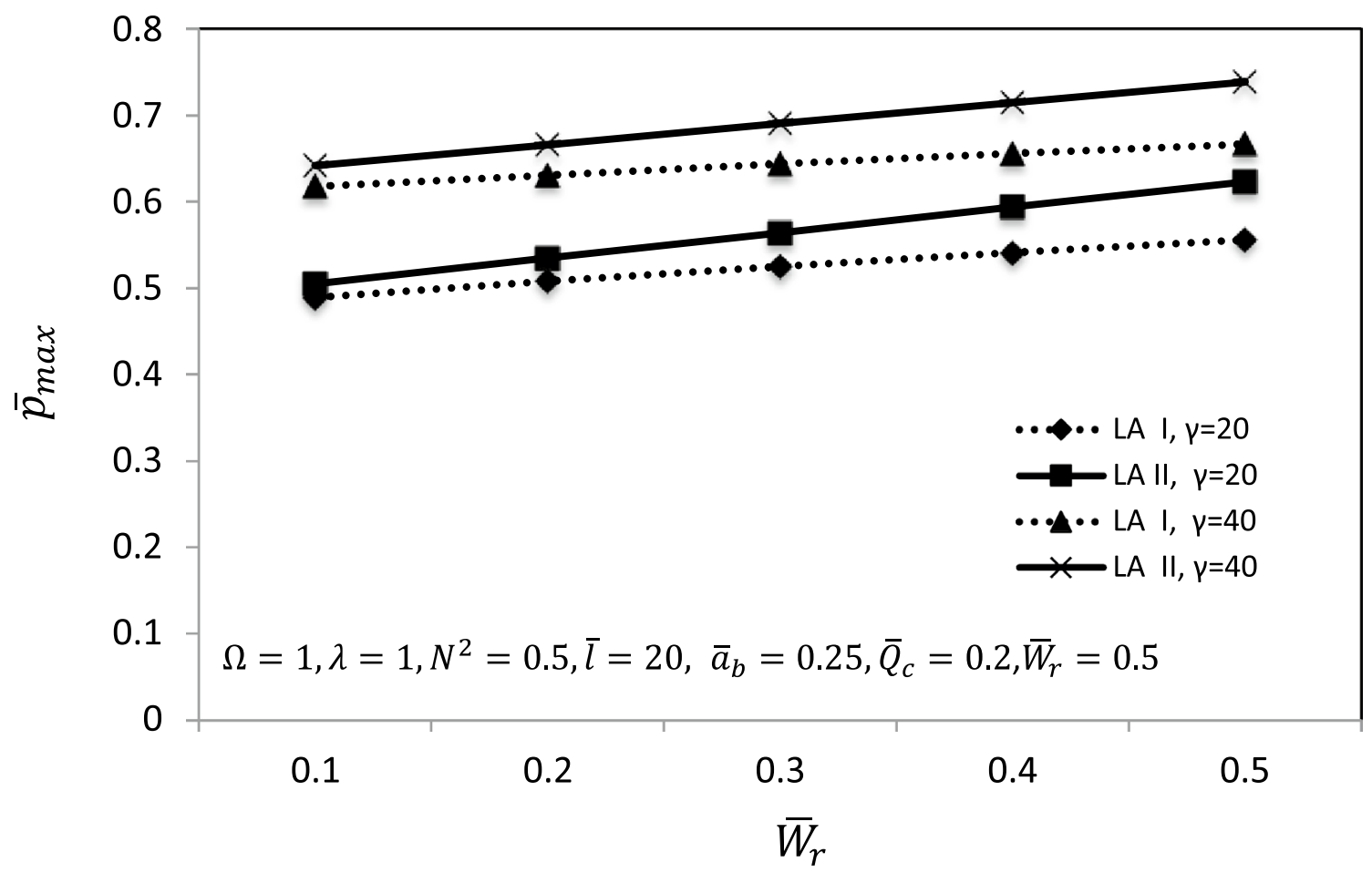

Fig. 5 Maximum fluid pressure $\left(\bar{p}_{\text {max }}\right)$ versus radial load $\left(\bar{W}_{r}\right)$ 


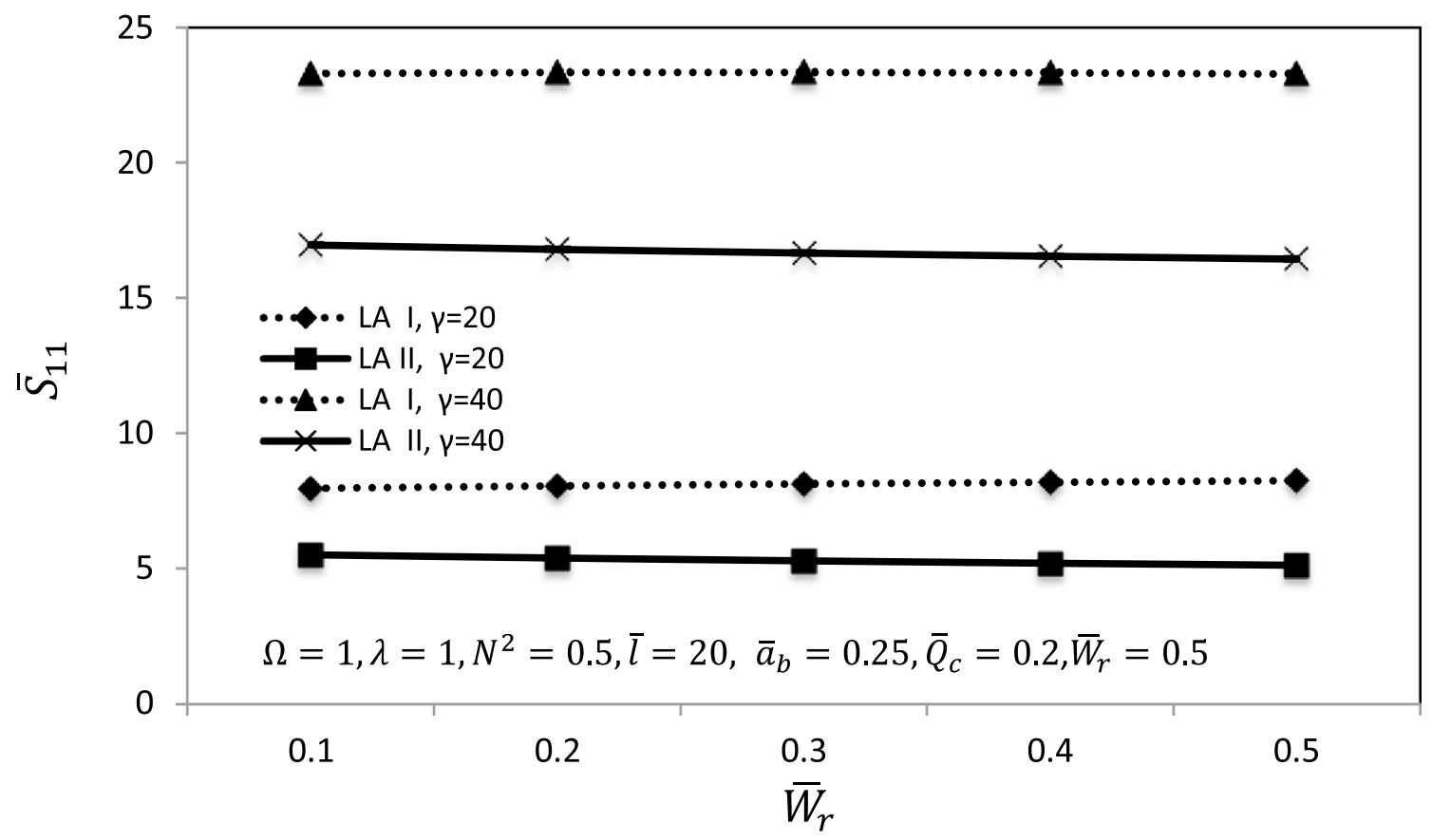

Fig. 6 Direct stiffness $\left(\bar{S}_{11}\right)$ versus radial load $\left(\bar{W}_{r}\right)$

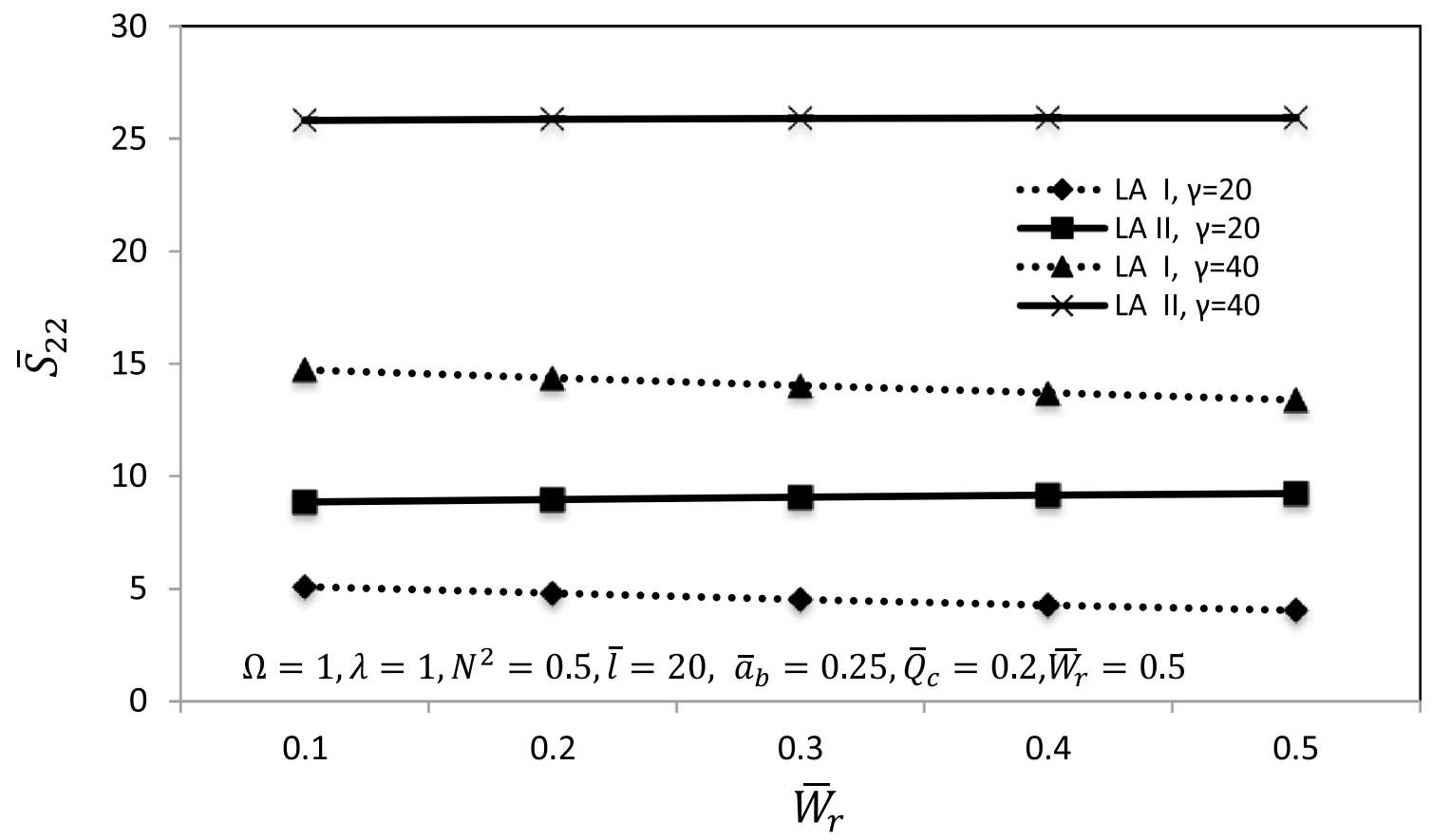

Fig. 7 Direct stiffness $\left(\bar{S}_{22}\right)$ versus radial load $\left(\bar{W}_{r}\right)$

variation of stiffness coefficients $\bar{S}_{11}$ and $\bar{S}_{22}$ compliment each other for both the loading arrangements LA I and LA II respectively.
The direct damping coefficients $\bar{C}_{11}$ and $\bar{C}_{22}$ versus $\bar{W}_{r}$ are plotted in Figs. 8 and 9. Both the damping coefficients $\bar{C}_{11}$ and $\bar{C}_{22}$ vary marginally with an increase in $\bar{W}_{r}$ for LA I and LA II for different $\gamma$. However, $\bar{C}_{11}$ and $\bar{C}_{22}$ are found to 


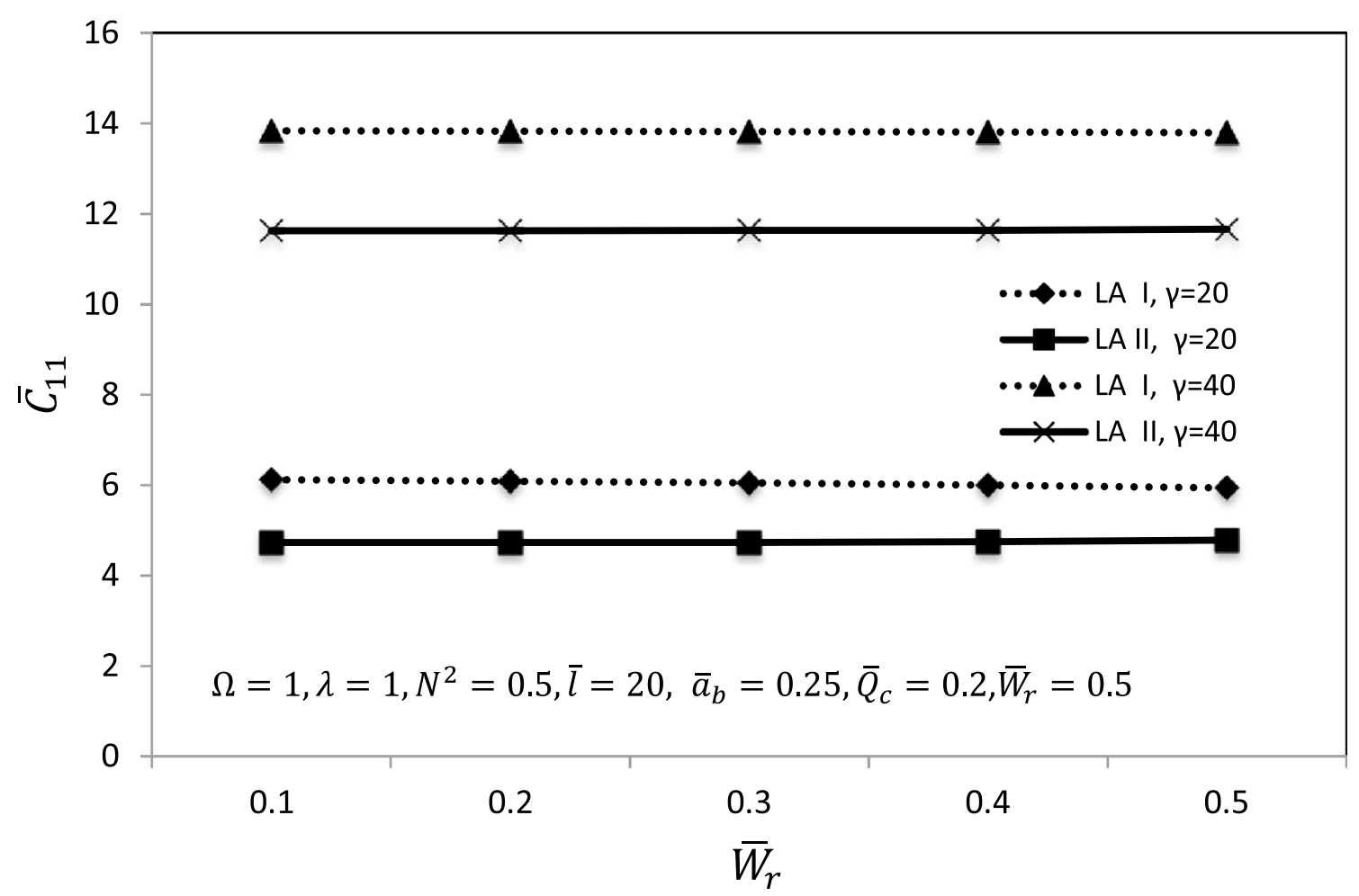

Fig. 8 Direct damping $\left(\bar{C}_{11}\right)$ versus radial load $\left(\bar{W}_{r}\right)$

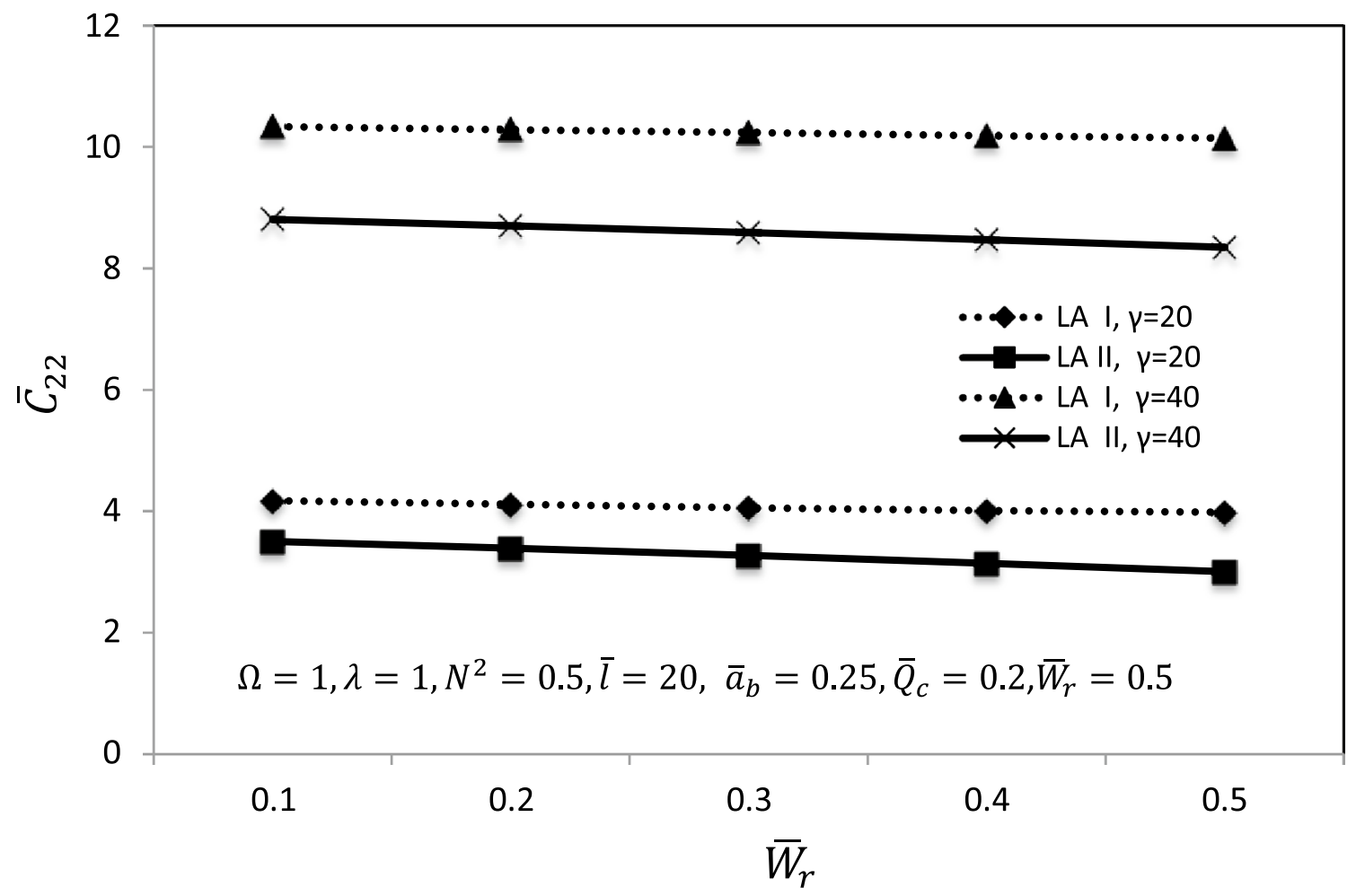

Fig. 9 Direct damping $\left(\bar{C}_{22}\right)$ versus radial load $\left(\bar{W}_{r}\right)$ 


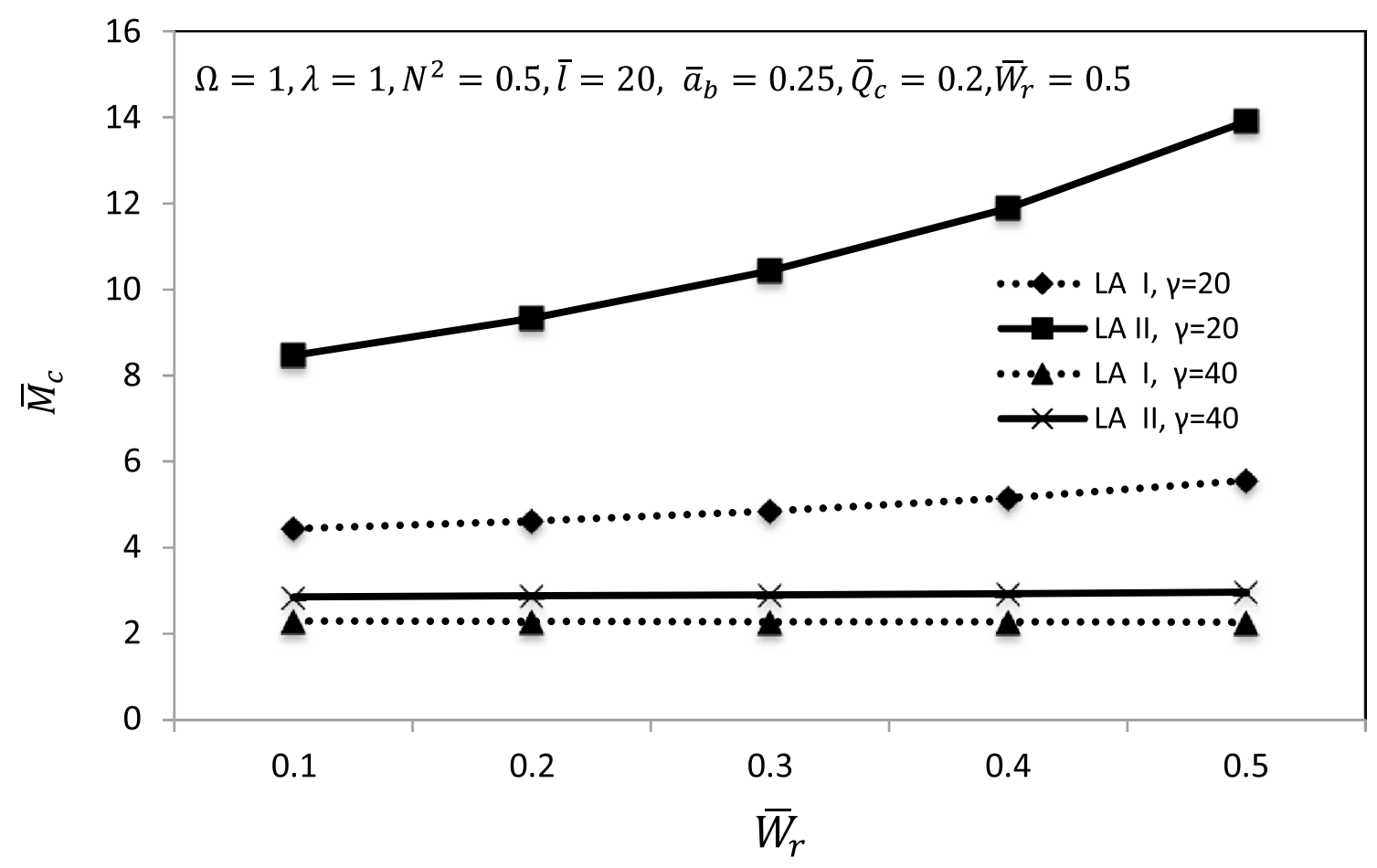

Fig. 10 Critical mass parameter $\left(\bar{M}_{c}\right)$ versus radial load $\left(\bar{W}_{r}\right)$

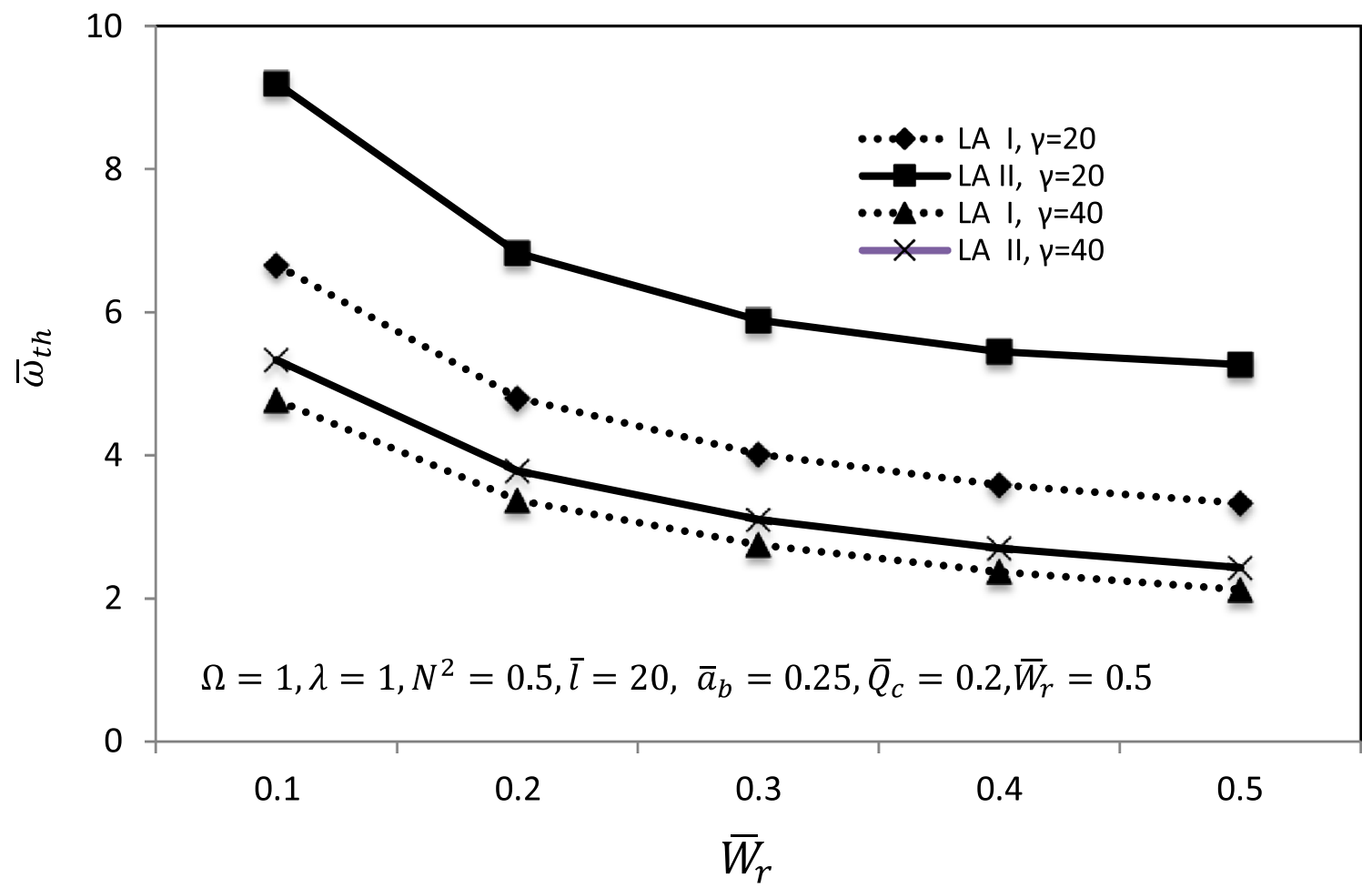

Fig. 11 Threshold frequency $\left(\bar{\omega}_{t h}\right)$ versus radial load $\left(\bar{W}_{r}\right)$ 
be increased for LA I than LA II position against $\bar{W}_{r}$. It can further be seen that for same amount of $\bar{Q}_{c}$ and $\bar{W}_{r}$, the value of $\bar{h}_{\min }$ is quite high for LA I than LA II and can play a significant role in the calculation of stiffness and damping coefficients.

\subsubsection{Influence on stability parameters}

The plots of critical mass parameter and threshold speed at $\gamma=20^{\circ}$ and $\gamma=40^{\circ}$ for both the loading conditions are presented in Figs. 10 and 11. It is found that at $\gamma=40^{\circ}$ both the stability parameters show the similar trend and are seen to be very close for LA I \& LA II. Whereas, at $\gamma=20^{\circ}$ the stability parameters are found to be higher when compared to $\gamma=40^{\circ}$. Hence, a combination of $\gamma=40^{\circ}$ with LA I can be considered from stiffness and damping coefficients point of view and $\gamma=20^{\circ}$ with LA II may be chosen from stability point of view.

\section{Conclusions}

The following conclusions can be drawn from the performance data obtained through present work that may be useful for the design of CFV compensated 4-pocket conical hybrid journal bearings:
1. The $\bar{h}_{\min }$ is found to be higher for LA I for a given range of the semi cone angle $\gamma$. Therefore, LAI configuration can be more useful than conventional configuration LA II from fluid film thickness point of view.

2. The stiffness coefficients $\left(\bar{S}_{11}\right)$ and $\left(\bar{S}_{22}\right)$ compliment each other for both the loading arrangements.

3. The damping coefficients $\left(\bar{C}_{11}, \bar{C}_{22}\right)$ are found to be higher for LA I load arrangement as compared to LA II.

4. The stability parameters for $\gamma=40^{\circ}$ with LA I arrangement may be useful from stiffness and damping coefficients point of view and $\gamma=20^{\circ}$ with LA II may be chosen from stability point of view.

\section{Compliance with ethical standards}

Conflict of interest The authors declare that they have no conflict of interest.

\section{Appendix}

See Fig. 12. 
Fig. 12 Solution scheme

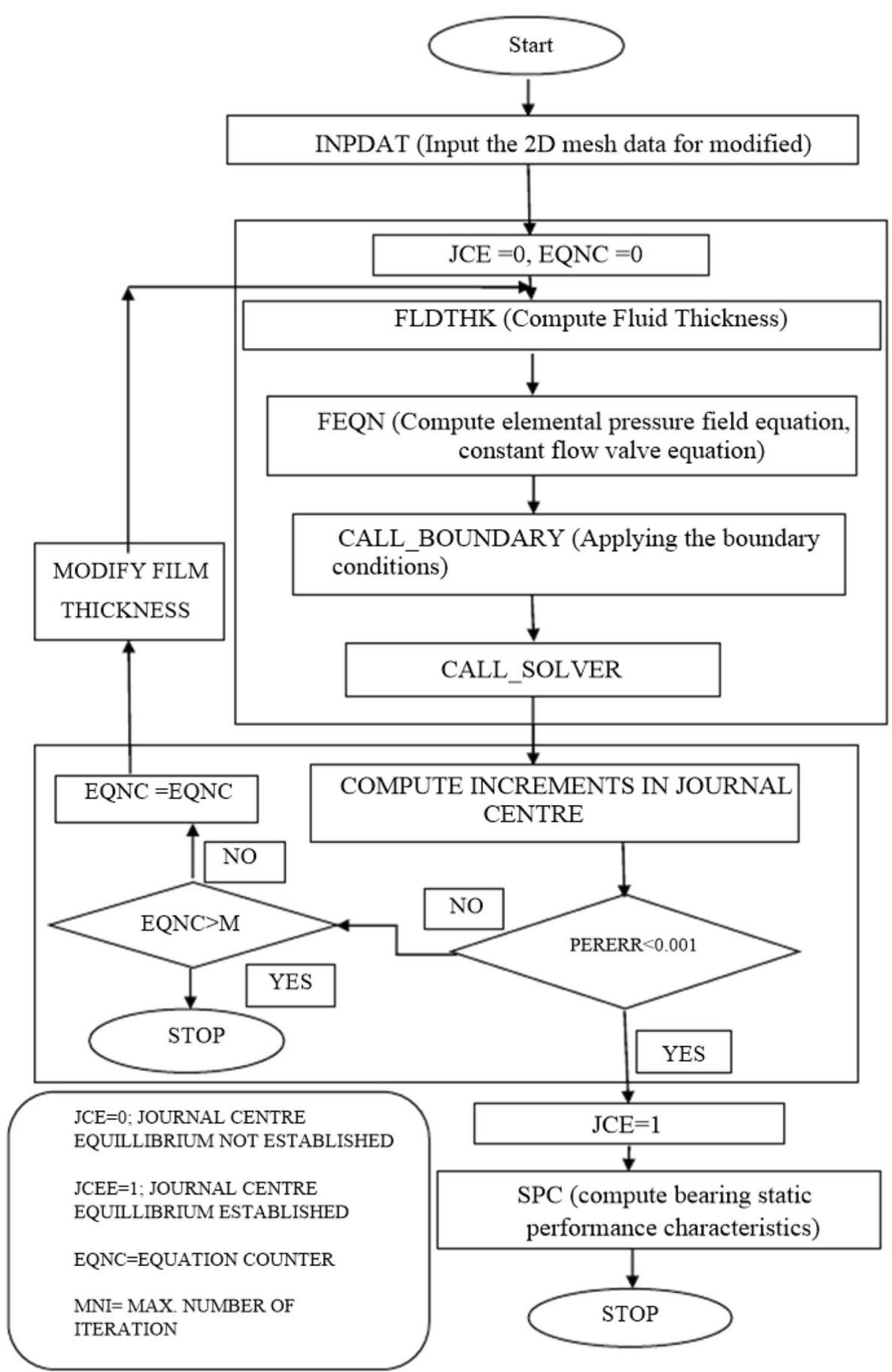

\section{References}

1. Verma S, Kumar V, Gupta KD (2009) Analysis of multirecess hydrostatic journal bearing operating with micropolar lubricant. J Tribol 131:021103-1

2. Rana NK, Gautam SS, Verma S (2014) Static characteristics of conical hydrostatic journal bearing under micropolar lubrication. J Inst Eng India Ser C 95(4):375-381

3. Rana NK, Gautam SS, Verma S, Rahmani F (2016) On the stiffness and damping coefficients of constant flow valve compensated conical hydrostatic journal bearing with micropolar lubricant. Procedia Technol 23:42-50

4. Nicodemus ER, Sharma SC (2010) Influence of wear on the performance of multirecess hydrostatic journal bearing operating with micropolar lubricant. J Tribol 132:021703-1

5. Sharma SC, Phalle VM, Jain SC (2011) Influence of wear on performance of a multirecess conical hybrid journal bearing compensated with orifice restrictor. Tribol Int 44:1754-1764

6. Rajput AK, Sharma SC (2013) Analysis of externally pressurized multirecess conical hybrid journal bearing system using micropolar lubricant. J Eng Tribol 227(9):943-961 
7. Rajput AK, Sharma SC (2014) Stability of a constant flow valve compensated multirecess conical hybrid journal bearing operating with micropolar lubricant. Lubr Sci 26(5):347-362

8. Dhawan R, Verma S (2014) Analysing micropolar lubrication in non-circular hybrid journal bearings. Tribol Trans 57:182-189

9. Sharma SC, Sinhasan R, Jain SC (1992) Performance characteristics of multirecess hydrostatic/hybrid flexible journal bearing with membrane type variable-flow restrictor as compensating device. Wear 152:279-300

10. Khakse PG, Phalle VM, Mantha SS (2016) Performance analysis of a non-recessed hybrid conical journal bearing compensated with capillary restrictors. J Tribol 138:011703-1

11. Guo H, Lai XM, Cen SQ (2009) Theoretical and experimental study on dynamic coefficients and stability for a hydrostatic/ hydrodynamic conical bearing. J Tribol 131:1-7

12. Zuo X, Wang J, Yin Z, Li S (2013) Comparative performance analysis of conical hydrostatic bearings compensated by variable slot and fixed slot. Tribol Int 66:83-92
13. Chen C, Kang Y, Huang C (2004) The influences of orifice restriction and journal eccentricity on the stability of the rigid rotorhybrid bearing system. Tribol Int 37:227-234

14. Rana NK, Gautam SS, Verma S (2016) Performance characteristics of constant flow valve compensated conical multirecess hybrid journal bearing under micropolar lubrication. Int J Des Eng 6(3):218-236

15. Rana NK, Gautam SS, Verma S (2016) Comparative study on the effect of recesses on conical hybrid journal bearing compensated with CFV under micropolar fluid lubrication. Tribol Online 11(3):474-486

16. Bhattacharya A, Dutt JK, Pandey RK (2017) Influence of hydrodynamic journal bearings with multiple slip zones on rotordynamic behavior. J Tribol 139:061701-1-11

Publisher's Note Springer Nature remains neutral with regard to jurisdictional claims in published maps and institutional affiliations. 